VOL. I3 (1975), 13-19.

\title{
A comparison theorem and the forced oscillation
}

\section{Hiroshi Onose}

Consider the $n$-th order delay differential equation

$$
x^{(n)}(t)+f\left(t, \tilde{x}\left(\tilde{g}_{0}(t)\right)\right)=q(t) .
$$

In the last few years, the oscillatory behavior of delay differential equations has been the subject of intensive investigations. But much less is known about the equation (A) with small forcing term $q(t)$. The only papers devoted to this problem are by Kartsatos, Kusano, and the present author. The purpose of this paper is to prove some new oscillation theorems which contain the previous results.

\section{Introduction}

We are concerned with the oscillatory behavior of solutions of the retarded differential equation

$$
x^{(n)}(t)+f\left(t, \tilde{x}\left(\tilde{g}_{0}(t)\right)\right)=q(t),
$$

where $\tilde{x}\left(\tilde{g}_{0}(t)\right) \equiv\left(x\left(g_{01}(t)\right), \ldots, x\left(g_{0(n-1)}(t)\right)\right)$

We only consider solutions of (A) which are defined and nontrivial for all large $t$. A solution $x(t)$ of (A) is called oscillatory if there exists a sequence $\left\{t_{m}\right\}_{m=1}^{\infty}$ such that $\underset{m \rightarrow \infty}{\lim } t_{m}=\infty$ and $x\left(t_{m}\right)=0$ for all $m$. Otherwise the solution is called nonoscillatory. Equation (A) itself

Received 5 March 1975. 
is called oscillatory if all solutions of (A) are oscillatory. $R^{d}$ denotes the $d$-dimensional space of vectors $\tilde{x}=\left(x_{1}, \ldots, x_{d}\right)$. The zero vector in $R^{d}$ is denoted by $\tilde{0}$. Inequality between vectors $\tilde{x}$ and $\tilde{y}=\left(y_{1}, \ldots, y_{d}\right)$ is defined as $\tilde{x} \geq \tilde{y} \quad[\tilde{x}>\tilde{y}]$ if and only if $x_{j} \geq y_{j}\left[x_{j}>y_{j}\right]$ for $j=1, \ldots, d$.

\section{Comparison theorem}

First we mention Kiguradze's [2] result:

LEMMA. If $x(t) \in C^{n}[a, \infty), x(t) \geq 0$ and $x^{(n)}(t)$ is nonnegative on $[a, \infty)$, then either

(I) $x^{\prime}(t), \ldots, x^{(n-1)}(t)$ tend monotonically to zero as $t \rightarrow \infty ;$ or

(II) there exists an odd integer $k, 1 \leq k \leq n-1$, for $n$ even and an even integer $k$ for $n$ odd, such that

$$
\begin{aligned}
& \lim _{t \rightarrow \infty} x^{(n-j)}(t)=0 \text { for } 1 \leq j \leq k-1, \lim _{t \rightarrow \infty} x^{(n-k)}(t) \geq 0, \\
& \lim _{t \rightarrow \infty} x^{(n-k-1)}(t)>0 \text { and } x(t), x^{\prime}(t), \ldots, x^{(n-k-2)}(t) \\
& \text { tend to } \infty \text { as } t \rightarrow \infty .
\end{aligned}
$$

For simplicity, we mention the following condition:

(a) $f\left(t, \tilde{y}_{0}\right)>\tilde{0}$ for $\tilde{y}_{0}>\tilde{0}, f\left(t, \tilde{y}_{0}\right)<\tilde{0}$ for $\tilde{y}_{0}<\tilde{0}$,

$$
\begin{aligned}
& f\left(t, \tilde{y}_{0}\right) \geq f\left(t, \tilde{z}_{0}\right) \text { for } \tilde{y}_{0} \geq \tilde{z}_{0} \geq \tilde{0}, \\
& g_{0 j}(t) \in c[[0, \infty), R], g_{0 j}(t) \leq t, \lim _{t \rightarrow \infty} g_{0 j}(t)=\infty,
\end{aligned}
$$

$j=1, \ldots, n-1, q(t)$ is continuous, and $f(t, \tilde{x})$ is continuous.

THEOREM 2. Consider the equation

$$
x^{(n)}(t)+f\left(t, \tilde{x}\left(\tilde{g}_{0}(t)\right)\right)=0
$$

and the inequality

$$
x^{(n)}(t)+f\left(t, \tilde{x}\left(\tilde{g}_{0}(t)\right)\right) \leq 0 .
$$


Assume that (a) holds. If, for $n$ even, equation (1) is oscillatory, then inequality (2) is oscillatory, while for $n$ odd, if every solution $x(t)$ of equation ( 1 ) is oscillatory, or $\lim x(t)=0$, then every solution of inequality (2) is oscillatory, or $\lim _{t \rightarrow \infty} x(t)=0$.

Proof. Suppose that there exists a nonoscillatory solution $x(t)$ of (2). Without loss of generality we may assume that $x(t)>0$ for $t \geq t_{0}$, where $t_{0}$ is a sufficiently large constant. Suppose first $n$ to be even. From (a), there is a $t_{1} \geq t_{0}$ such that $x\left(g_{0 j}(t)\right)>0$ $(j=1, \ldots, n-1)$ for $t \geq t_{1}$. From (2), we have

$$
x^{(n)}(t)=-f\left(t, \tilde{x}\left(\tilde{g}_{0}(t)\right)\right)<0 .
$$

By repeated integration of (3), we 'obtain

(4) $x(t) \geq c+\int_{t_{2}}^{t} \int_{t_{2}}^{u_{n-1}} \ldots \int_{t_{2}}^{u_{n-l+1}} \int_{u_{n-l}}^{\infty} \ldots$

$$
\begin{gathered}
\ldots \int_{u_{1}}^{\infty} f\left(s, \tilde{x}\left(\tilde{g}_{0}(s)\right)\right) d s d u_{1} \ldots d u_{n-1} \\
\equiv c+\phi\left(t, \tilde{x}\left(\tilde{g}_{0}(t)\right)\right) \text {, for } t \geq t_{2},
\end{gathered}
$$

where $c$ is a positive constant and $l$ is the constant of the lemma.

Now consider the equation

$$
z(t)=\phi\left(t, \tilde{z}\left(\tilde{g}_{0}(t)\right)\right)+c .
$$

If (5) has a solution $z(t)>0$ for $t \geq t_{2}$, then $z(t)$ is a positive solution of (1). To prove this, we define $\left\{z_{n}(t), n=0,1, \ldots\right\}$ such that

$$
\begin{aligned}
& z_{0}(t)=x(t) \quad \text { for } t \geq t_{1} \text {, } \\
& z_{n+1}(t)=\left\{\begin{array}{ll}
c+\phi\left(t, \tilde{z}_{n}\left(\tilde{g}_{0}(t)\right)\right) & \text { for } t \geq t_{2}, \\
c & \text { for } t_{1} \leq t \leq t_{2} .
\end{array} .\right.
\end{aligned}
$$


Then we see that $z_{n}(t)$ is well-defined, and

$$
0<z_{n}(t)<x(t), c \leq z_{n+1}(t) \leq z_{n}(t) \text {. }
$$

If we put

$$
z(t)=\lim _{n \rightarrow \infty} z_{n}(t) \text {, for every point of } t\left(\geq t_{1}\right) \text {, }
$$

then by (6), (7), and Lebesgue's Theorem, we have

$$
z(t)=c+\phi\left(t, \tilde{z}\left(\tilde{g}_{0}(t)\right)\right) \text {, for all } t \geq t_{2} \text {. }
$$

Hence we see that ( 1 ) has a nonoscillatory solution. Suppose $n$ is odd. By the lemma, if $x^{\prime}(t)>0$, then the proof proceeds as in the even case. In case $x^{\prime}(t)<0$ and $\lim _{t \rightarrow \infty} x(t) \neq 0$, put $L=\lim _{t \rightarrow \infty} x(t)$ and consider the following inequality

$$
x(t) \geq L / 2+\int_{t}^{\infty} \int_{u_{n-1}}^{\infty} \ldots \int_{u_{1}}^{\infty} f\left(s, \tilde{x}\left(\tilde{g}_{0}(s)\right)\right) d s d u_{1} \ldots d u_{n-1} .
$$

Then the rest of the proof is the same as in the case of even $n . / /$ REMARK. Theorem 1 shows that it is sufficient to consider only equation (1) for the oscillation theorem.

\section{Forced oscillation}

THEOREM 2. Consider the equations

$$
x^{(n)}(t)+f\left(t, \tilde{x}\left(\tilde{g}_{0}(t)\right)\right)=0
$$

and

$$
x^{(n)}(t)+f\left(t, \tilde{x}\left(\tilde{g}_{0}(t)\right)\right)=q(t) .
$$

Assume that (a) and the following condition hold:

(10) there exists $Q(t)$ such that $Q^{(n)}(t)=q(t), \lim _{t \rightarrow \infty} Q(t)=0$, and $Q(t)$ has an unbounded set of zeros.

If, for $n$ even, (8) is oscillatory, then (9) is oscillatory, while for $n$ 
odd, if every solution $x(t)$ of (8) is oscillatory, or $\lim _{t \rightarrow \infty} x(t)=0$, then every solution $x(t)$ of (9) is oscillatory, or $\lim _{t \rightarrow \infty} x(t)=0$.

Proof. Let $x(t)$ be a nonoscillatory solution of (9) and assume $x(t)>0$ for $t \geq t_{0}$. Put $y(t) \equiv x(t)-Q(t)$; then $y(t)$ satisfies

(II) $y^{(n)}(t)+f\left(t, y\left(g_{01}\right)+Q\left(g_{01}\right), \ldots, y\left(g_{0(n-1)}\right)+Q\left(g_{0(n-1)}\right)\right)=0$.

From (11), we see $y^{(n)}(t)<0$, so that we have

$$
y^{\prime}(t) \text { and } y(t) \text { are of constant sign. }
$$

If we suppose $y(t)<0$, then we have $0<x(t)<Q(t)$; this leads to a contradiction to the assumption that $Q(t)$ has an unbounded set of zeros. So we have

$$
y(t)>0 \text {. }
$$

From (12) and (13), we have that $y^{\prime}(t)<0$ can happen only for $n$ odd. By (12), there exists a definite limit of $y(t)$,

$$
\lim _{t \rightarrow \infty} y(t)=c, \quad(0 \leq c \leq \infty),
$$

where $c=0$ can only happen for the case $n$ odd. For $c=0$, we have that $\lim x(t)=0$. For $c>0$, put $z(t) \equiv y(t)-c / 2$; then we have

$$
0<c / 2<z(t)<y(t)+Q(t) \text {, for } t \geq t_{2}>t_{1} \text {. }
$$

From (11) and (15) we have

$$
z^{(n)}(t)+f\left(t, \tilde{z}\left(\tilde{g}_{0}(t)\right)\right) \leq 0 \text { for } t \geq t_{3}>t_{2} .
$$

Now we consider the equation

$$
z^{(n)}(t)+f\left(t, \tilde{z}\left(g_{0}(t)\right)\right)=0 \text { for } t \geq t_{3} .
$$

Since equation (16) has a solution $z(t)$ such that $\lim _{t \rightarrow \infty} \inf z(t)>c / 2>0$ we see, from Theorem 1 , that not every solution $z(t)$ of (17) is oscillatory or $\lim _{t \rightarrow \infty} z(t)=0 . \quad / /$ 
THEOREM 3. Assume that (a) and the following condition hold:

(10') there exist $Q(t)$ such that $Q^{(n)}(t)=q(t)$ and $\lim Q(t)=0$.

$t \rightarrow \infty$

For $n$ even, if (8) is oscillatory, then every solution $x(t)$ of (9) is oscillatory or $\lim x(t)=0$. For $n$ odd, if every solution $x(t)$ of (8) is oscizlatory or $\lim _{t \rightarrow \infty} x(t)=0$, then every solution $x(t)$ of (9) is oscizlatory or $\lim _{t \rightarrow \infty} x(t)=0$.

Proof. The proof is contained in the proof of Theorem 2.

THEOREM 4. Assume that (a) and the following condition hold:

(18) there exist constants $q_{1}, q_{2}$ and sequences $\left\{t_{s}^{\prime}\right\},\left\{t_{s}^{\prime \prime}\right\}$ such that $\lim _{t \rightarrow \infty} t_{s}^{\prime}=\lim _{t \rightarrow \infty} t_{s}^{\prime \prime}=\infty, Q\left(t_{s}^{\prime}\right)=q_{1}, Q\left(t_{s}^{\prime \prime}\right)=q_{2}$ and $q_{1} \leq Q(t) \leq q_{2}$

For $n$ even, if (8) is oscillatory, then every solution of (9) is oscillatory, while for $n$ odd, if every solution $x(t)$ of (8) is oscillatory or Iim $x(t)=0$, then every solution of (9) is oscillatory or $\lim _{t \rightarrow \infty}(x(t)-Q(t))=-q_{1} \quad$ (or $\left.-q_{2}\right)$.

Proof. The same procedure of the proof of Theorem 2 is used. Suppose that $x(t)>0$ is a nonoscillatory solution of (9). Put $y(t) \equiv x(t)-Q(t)$. Then we see that our proof proceeds until (12). Put $\boldsymbol{z}(t)=y(t)+q_{1}$. Then, by the fact that $y^{\prime}(t)$ is monotone, we have

$$
\lim _{t \rightarrow \infty} z(t)=c \quad(-\infty \leq c \leq+\infty) .
$$

If $c$ is negative, then we have $y(t)+q_{1}<0$ for sufficiently large

$t$; this leads to a contradiction to the fact that

$$
y\left(t_{s}\right)+q_{1}=y\left(t_{s}\right)+Q\left(t_{s}\right)=x\left(t_{s}\right)>\dot{0} .
$$

If $c=0$, then we have that $\lim _{t \rightarrow \infty}(x(t)-Q(t))=-q_{I}$. If $c$ is positive, 
then we have

$$
0<z(t)=y(t)+q_{1} \leq y(t)+Q(t) .
$$

By (11) and (20), we have

$$
z^{(n)}(t)+f\left(t, \tilde{z}\left(\tilde{g}_{0}(t)\right)\right) \leq 0 \text { for } t \geq t_{2} .
$$

Here, we consider the following equation

$$
z^{(n)}(t)+f\left(t, \tilde{z}\left(\tilde{g}_{0}(t)\right)\right)=0 \text { for } t \geq t_{2} .
$$

From (21), (22), and Theorem 1, we have the proof.

REMARK. The results of Kartsatos [1] and Kusano and the present author [3] are contained in Theorem 2, Theorem 3, and Theorem 4.

\section{References}

[1] Athanassios G. Kartsatos, "Maintenance of oscillations under the effect of a periodic forcing term", Proc. Amer. Math. Soc. 33 (1972), 377-383.

[2] Н.T. Ннгурадзе [1.T. Kiguradze], "Н вопросу нолеблемости решений нвлннейных дифференцнальных уравненнй" [The problem of oscillation of solutions of nonlinear differential equations], Differencial'nye Uravnenija 1 (1965), 995-1006; Differential Equations I (1965), 773-782.

[3] T. Kusano and H. Onose, "Oscillations of functional differential equations with retarded argument", J. Differential Equations 15 (1974), 269-277.

Department of Mathematics, Ibaraki University, Mito, Japan. 